\title{
Chaotic laser brings out higher precision OTDR
}

\author{
WANG YunCai
}

Professor Wang YunCai and his student Wang AnBang reported a new concept of optical time domain reflectometry (OTDR) based on a chaotic light correlation method. This will be useful for precise fault location in fiber links with high-density events. Their work is reported in Issue 53 (February, 2010) of SCIENCE CHINA Information Sciences.

Optical fiber cables often break down due to natural disasters or human destruction. In particular, there are more and more fiber cable breakdowns within a building with the development of local networks and radio over fiber technology. This problem is currently solved by OTDR, the most important tool for fiber fault location. However, the conventional one, based on the pulse-echo method, is not precise enough because of its several meters of spatial resolution and its broad dead zone.

By the use of a chaotic light correlation technique, Dr. Wang and Prof. Wang achieved distance-independent resolutions of $6 \mathrm{~cm}$ and $25 \mathrm{~km}$ measurable distance for single-mode fiber Although the correlation technique was used in a pseudo-random sequence OTDR to improve the trade-off between resolution and dynamic range in a pulse-echo OTDR, it is still limited by the electronic modulation bandwidth. The use of broadband chaotic light generated from a laser diode rather than pseudo-random optical pulses, reached centimeter accuracy for fiber fault location. Its obvious advantage is that a laser diode with optical feedback can easily generate large-amplitudes and multi-GHz bandwidth chaotic light signal, without electro-optical modulator.

"This paper reports the first chaotic OTDR experiments, which will be useful for evaluation of optical fibers", said one of reviewers. After Prof. Wang introduced their work at the CHAOS 2009 International Conference held in Chania, Greece (1-5 June, 2009), the chaotic correlation OTDR was considered to be one of three novel applications according to the summary of the international workshop of Chaos Applications in Telecommunications and Sensors, 2009 (The other two are fast pseudorandom bit generation and chaos steganography, by the way).

"The current research emphasizes the high accuracy of fiber fault location and the next aim is to measure the fiber loss and Rayleigh scatters just as the conventional correlation OTDR does", said Prof. Wang. The achievement of a chaotic correlation OTDR makes it possible for high-accuracy and non-destructive detection of small-size fiber networks.

The authors are affiliated to the Laboratory of Optical Information and Technology, Physics Department, Taiyuan University of Technology. This laboratory is doing research mainly in the fields of nonlinear dynamics in lasers and applications, optical communications and sensors, and fast random number generation.

This work was supported by the National Natural Science Foundation of China (Grants Nos. 60908014 and 60777041$)$ and the International Cooperation Project of Shanxi Province, China (Grant No. 2007081019).

See the article: Wang A B, Wang Y C. Chaos correlation optical time domain reflectometry. Sci China Inf Sci, 2010, 53: 398-404, doi: 10.1007/ s11432-010-0038-4 